\title{
Adolescents' Literacy on Contraceptive Usage and Their Right towards Healthy Living in Ekiti State, Nigeria.
}

\author{
Dr. Mrs. E. O. Osakinle, Dr. J.O. Babatunde, Dr. Mrs. F.A. Alade \\ Dr. F.O. Bada, Dr. B.A. Falana, Dr. Mrs. G.F. Olusegun \\ Faculty Of Education,Ekiti State University, Ekiti State, Nigeria.
}

\begin{abstract}
Adolescents are the groups of individuals that are prone to all forms of risks because of the stage they are in. They need to be educated as they take a lot of risks concerning their health and living right. Reproductive health is about the healthy and well being of people of all ages. However, because of the age and being unmarried, most of the adolescents do not usually have access to reproductive health facilities in their different locations as do the adults. Also, their culture of keeping their secrets to themselves make them become sick and morbid from various communicable diseases they had contracted. Since adolescents make up $20 \%$ of the world's population and about $85 \%$ live in developing countries, their education on sexual and reproductive health need to be looked into.
\end{abstract}

Keywords: Adolescents, Sexual Rights, Reproductive Health

\section{Introduction}

Adolescence is the range when persons are 10-19 years old. Adolescents make up approximately $20 \%$ of the world's population and about $85 \%$ live in developing countries (Osakinle, 2003; Osakinle \& Aladegbola 2012). The concept of reproductive health is almost twenty years old. The international Conference on Population and Development (ICPD) in 1994 marked the birth of this concept, defining reproductive health as "a state of complete physical, mental and social well-being and not merely the absence of disease or infirmity in all matters related to the reproductive tract, its functions and processes (Obaid, 2009).

Sexual health was originally defined as one of the components of reproductive health but over time, the concept of reproductive health has come to be known as sexual and reproductive health (SRH) covers issues such as education, gender, poverty and mobility in addition to the original components of reproductive health (Shaw, 2009).

\section{Problem of Adolescents' Access to Reproductive Health}

Due to controversies related to adolescent sexuality and the general lack of knowledge about the reproductive and sexual needs of adolescents, very few countries in the world have set up adequate reproductive health care services for adolescents (The Alan Gutt. Inst (1998). Adolescent reproductive health care needs vary with culture, age, and marital status. But all adolescents need accurate and adequate information about sexual and reproductive health. They also require accessible and affordable reproductive health services. Without easy access to accurate information, adolescents are at risk of being misinformed about sexual and reproductive matters, which may lead them to make decisions that could have negative effects on their lives. Moreover, adolescents need information about safe-sex practices, including negotiation skills to protect them from potentially dangerous and abusive relationships. Since pregnant adolescents face greater risk of complications than adult women, adolescents' access to quality and affordable prenatal care is critical (The Alan Gutt. Inst (1998; Osakinle, 2003).

Adolescents are also concerned about privacy and confidentiality regarding reproductive health care. This is particularly important for unmarried adolescents who confront negative attitudes for being sexually active. Such attitudes only serve to alienate adolescents from seeking reproductive health care. The same adolescents also require access to contraception to protect themselves from unwanted pregnancies and sexually transmissible infections, including HIV (Osakinle, 2003).

\section{Education And Adolescents}

A key condition to fulfilling the reproductive rights of adolescents is education. Education enables adolescents to obtain information that they can use to exercise and protect a range of interests and rights, including their reproductive rights. Studies have shown that around the world, across different regions and cultures, educated women have a greater say in their reproductive lives than women who have little or no education. These studies also indicated that a minimum of five years of education is required to enable a woman to control her reproductive life. An educated adolescent is more likely to seek reproductive health information 
and services than an uneducated one, moreover, education increases women's self confidence and self-esteem, employment opportunities, and ability to provide for themselves.

Low school attendance of girls is related primarily to gender and lack of economic resources. With regard to gender, in societies where early marriage is the norm, adolescent girls are often withdrawn from school to get married. Also, in several countries, adolescent girls who get pregnant are expelled from school. In many rural areas, families cannot afford to send all their children to school, and it is often the daughters' education that is sacrificed (The Alan Gutt. Inst (1998). Along with formal education, it is equally important to provide adolescents and girls with education about sexual and reproductive matters. Many countries resist such education in a formal setting under the erroneous assumption that educating adolescents about sexuality will encourage early sexual activity. However, studies have shown that sex education actually has the opposite effect of delaying sexual activity (State of World Population; 1997).

\section{Why are sexual and reproductive health and rights important?}

Sexual and reproductive health is an essential element of good health and human development. Sexual and reproductive health is also important as an issue in itself. People have right to make their own choices and decisions, based on sound information. Reproductive rights must be protected, promoted and fulfilled if sexual and reproductive health outcomes are to be improved, particularly for the poor and vulnerable, empowering people to take their own decisions about sexual and reproductive lives and ability of poor and vulnerable people to demand and use services and information and to be heard, it also puts emphasis on equitable access to services and women's empowerment.

\section{What do we mean by sexual and reproductive health and rights?}

International Conference on Population Development (ICPD) defined reproductive health as "a state of complete physical, mental and social well-being and not merely the absence of disease or infirmity, in all matters relating to the reproductive system and to its functions and processes". Men and women should be able to enjoy a satisfying and safe sex life, have the capability to reproduce and the freedom to decide it, when and how often to do so. This requires informed choices and access to safe, effective, affordable and acceptable health-care services.

International Conference on Population Development (ICPD) defined reproductive health care as "the constellation of methods, techniques and services that contribute to reproductive and sexual health and wellbeing by preventing and solving reproductive health problems. It also includes sexual health, the purpose of which is the enhancement of life and personal relations and not merely counselling and care related to reproductive and sexually transmitted diseases".

Reproductive rights are defined in the ICPD Programme of Action paragraph 7.3, and are based upon rights recognized in international human right treaties, declarations and other instruments, including the International Covenant on Economic, Social and Cultural Rights, the International Covenant on Civil and Political Rights, the UN Convention on the Elimination of All Forms of Discrimination Against Women (CEDAW), the UN Convention on the Rights of the Child, and The 2004 UN Commission on Human Rights explicitly recognized women's sexual rights as essential to combating violence and promoting gender equity; ICPD recognized that people's sexual and reproductive health needs are rights that they are entitled to demand. Specific rights relevant to sexual and reproductive health according to UNFPA (2004) and Cook, et al., (2003): the following are some of the rights:

- Right to the highest attainable standard of health

- $\quad$ Right to life and survival

- Right to liberty and security of person.

- Right to be free from torture, cruel, inhuman or degrading treatment.

- Right to decide freely and responsibly the number and spacing of one's children and to have information and means to do so.

- Right of women to have control over and decide freely and responsibly on matters related to their sexuality, including sexual and reproductive health, free to coercion, discrimination and violence.

- Right to privacy.

- Right to freedom from discrimination (on the basis of sex, gender, marital status, age, race, ethnicity, health status/disability).

- Right of access to information.

- Right to education.

- Right to freedom from violence against women. 


\section{Factors Influencing Sexual Activity Among Young People Include:}

- Earlier onset of sexual maturation and the accompanying natural increase in body secretions (sex hormones) which stimulates sexual urges in adolescent boys and girls.

- Pressure by the peer group and adults on young people to engages in sexual relations.

- Increasing socio-economic problems which results in pressure on young people to exchange sex for money.

- Glamorization of sex in the mass media without highlighting the associated risks.

- Permissive attitude of society towards premarital sexual relations for boys and girls as part of their predatory sexual socialization.

- Culture which places higher value on child-bearing as a greater achievement for girls.

- Parents who give their daughters in marriage as an early age for economic gains or under the guise of protecting her from herself or temptation from others.

- Delayed marriage for reasons of increasing focus on education/career pursuits. While marriage is being delayed, the other factors listed above combine together to influence sexual activity among young people.

The health and social consequences of early unwanted pregnancy include:

$\rightarrow$ Girls aged 10-14 years are five more times more likely to die in pregnancy or childbirth than women aged.

$\rightarrow$ Pregnancy-related complications are the main cause of death in 15-19 year old girls worldwide.

$\rightarrow$ Other health complications for mother and child include bleeding in pregnancy, severe anaemia, prolonged difficult and obstructed labour, still birth, low birth weight and infantile death.

$\rightarrow$ Socio-economic consequences for the young person may include termination of education, poor job prospects, loss of self-esteem and broken relationships.

Reasons why young girls continue to procure abortions include:

- Lack of accurate and comprehensive information about their sexual and reproductive health.

- Lack of appropriate reproductive health counselling and clinical services.

* Non-use or ineffective use of contraceptives by sexually active young people.

* Fear or rejection by partners, parents, peer group, religious and community leaders, once they find out about the pregnancy.

* Financial and emotional inability to care for a baby.

\section{Way Forward:}

- Accurate and comprehensive information on their sexuality;

- Support and skills to postpone starting sex for those who are not yet sexually active;

- Access to protective measures to prevent unwanted pregnancy, STDs including HIV/AIDS for those who have initiated sex;

- Protection and care from sexual abuse and exploitation; and

- Access to a range of youth-friendly services that are designed to promote their sexual and reproductive health.

\section{The Role of Parents/Guardians:}

Parents are the primary sexuality educators of their children. They educate both by what they say and by how they behave. It is important to begin deliberate education at the earliest childhood level; however, adolescence poses new challenges for many parents. In homes where there is open communication about sexuality, young people often behave more responsibly. At a minimum, such communication may help young people accept their own sexual feelings and actions. With open communication, young people are more likely to turn to their parents in times of trouble; without it, they will not.

\section{Specifically, Parents can:}

i. Make sure that they themselves are well-informed about human sexuality;

ii. Model sexuality healthy attitudes in their own relationships;

iii. Talk with their children about reproductive health and sexual responsibility and answer all the questions fully and accurately;

$i v$. Listen to their children compassionately, without dismissing their concerns as childish or condemning their questions as improper.

v. Provide a supportive and safe environment for their children as well as set and maintain limits for dating and other social activities they are involved in, outside the home. 
vi. Appeal for and support national, community and in-school efforts to provide young adult with reproductive health information and services.

\section{The Role of Teachers and Educational Administrators:}

Schools are an important site where young people can acquire knowledge and skills that equip them for responsible lifestyles now and in the future.

\section{Specifically, Teachers and Educators can:}

i. Actively support the development of school curricula that gives students age-appropriate comprehensive sexuality education;

ii. Train and support teachers so that they can teach about sexual and reproductive health accurately and comfortably.

iii. Ensure that school activities provide experiences that reinforce values and group norms against unprotected sexual behaviours;

iv. Facilitate better communication about sexuality and contraception between students and their parents by sensitizing parents on the need to show interest; and

v. Enhance positive social relationships between teachers and students as well as support Guidance counselors in schools to perform their expected roles.

\section{The Role of Health Care Provides:}

Health care organizations and providers have the responsibility to provide young people with affordable, sensitive and confidential sexual and reproductive health care services. As professionals, health provides can not afford to allow their personal or religious views becloud their obligations to assist young people who come to seek information and services.

\section{Specifically, leaders of reproductive health programmes can:}

i. Establish health care protocols that meet the needs of young adults;

ii. Be sensitive to the concerns of the community while acting as advocates for meeting young adults' needs;

iii. Involve young people in programme design, delivery and evaluation;

iv. Train health care providers to offer high-quality care to young adults in a nonjudgmental, confidential manner;

v. Make clear to staff and the public that young clients are welcome and that their care has high priority;

vi. Provide information and services at times and in ways that are acceptable and convenient for young adults.

\section{The Role of Policy Makers:}

Public policies on adolescent sexual health should be based on knowledge of adolescent development, accurate data, am established theoretical bases ford programme effectiveness, ongoing evaluation and adequate funding and support.

\section{Specifically, policy makers and political leaders can enact and enforce policies that:}

i. Improve young people's access to sexual and reproductive health information and services;

ii. Prohibit the abuse of young people, including sexual abuse and female genital cutting;

iii. Make public statements that emphasizes the importance of young people's reproductive health;

iv. Endorse and commit funding to support realistic programs to address young people's concerns;

v. Insist that the news and entertainment media provide more responsible coverage and treatment of sexual behaviours;

vi. Facilitate optional adolescent development by ensuring high quality education and employment opportunities for young people and involve them in programme planning and implementation.

\section{The Role of Religious and Community Leaders:}

Religious and Community Leaders are often called opinion leaders. They are highly respected by the people and have a responsibility to assist young people deal with reality by giving them a consistent set of messages regarding community values about such issues as sexual behaviours, responsibility and future planning. When these leaders understand and accept the importance of addressing young people's needs, it becomes easier for them to promote these issues among members of their community. 


\section{Specifically, religious and community leaders can:}

i. Urge understanding, compassion and concern for young people among their captive audiences and congregations;

ii. Make the community aware that there are social as well as personal causes of young people's reproductive health problems;

iii. Speak publicly to their congregations and others about young people's health needs and encourage them to support these needs;

iv. Offer young people support and guidance to explore and affirm their own values, as well as provide opportunities for them to benefit from mentoring by adult role models;

v. Initiate efforts to provide young people with reproductive health information and services; and

vi. Advocate and organize substantial reproductive health programmes to reach in and out of school youths.

\section{The Role of the Mass Media}

The mass media have become a major source of information about sexuality; mass media professionals can exercise their influence by providing accurate information and modeling responsible behaviours. The communication of accurate information adds realism and help adolescents gain insight into their own sexuality. By so doing, young people will be able to make more responsible decisions about their behavior.

\section{Specifically, mass media practitioners can:}

i. Give prominence to news events concerning the health of young people and make the public aware of young people's health needs;

ii. Provide air time and newspaper spaces for reports, spot announcements and jingles related to sexuality education for young people, at a low cost or free of charge;

iii. Strike a balance between the social responsibility of the media and financial expectation from adverts;

iv. Stop glamorizing and glorifying irresponsible sex. Portray scenes to emphasize that sexual encounters should be planned events, not spur-of-the-moment responses to the heat of passion

v. Emphasize the consequences of early sexual activity ands incorporate the prevention of pregnancy ands sexually transmitted diseases into drama scripts, news coverage and other informational formats; and

vi. Address parents with accurate information and guidance on talking with their adolescent children about sexuality and relationships.

\section{Conclusion}

The low levels of contraceptive use reflect the spontaneity of adolescent sexual activity and lack of knowledge, as well as many barriers young people face when they attempt to obtain contraceptive protection. The issue of discovering young people from obtaining professional birth control education and services place them at an increased risk of pregnancy and STDs including AIDs.

However, some sexually active adolescents practice contraception. Appreciable proportions lack knowledge about contraception and reproductive health. Parents and teachers are to be provided with the skills to socialize their children on sexual matters. This will go a long way in helping the adolescents in their reproductive health thereby living sexually healthy.

\section{Recommendations}

- Identify patients with limited literacy levels

- Use simple language, short sentences and define technical terms

- Supplement instruction with appropriate materials (videos, models, pictures etc.)

- Ask patients to explain your instructions (teach back method) or demonstrate the procedure

- Ask questions that begin with "how" and "what" rather than closed-ended yes/no questions

- Organize information so that the most important points stand out and repeat this information

- Reflect the age, cultural, ethnic and racial diversity of patients

- $\quad$ For Limited English Proficiency (LEP) patients, provide information in their primary language

- Improve the physical environment by using lots of universal symbols

- Offer assistance with completing forms

- Human rights awareness among the poor and vulnerable members 


\section{References}

[1]. Cook, R. J., Dickens, B. M., Fathalla, M. F. (2003): Reproductive Health and Human Rights; Integrating Medicine, Ethics and Law. Oxford: Oxford University Press.

[2]. Obaid, T. A. (2009): Fifteen years after the International Conference on Population and Development: What have we achieved and how do we move forward? Int. Journal of Gynaecol Obset, 2009; 106(2): 102-105.

[3]. Osakinle E.O. \& Aladegbola A.G. (2012): Effectiveness of Two Behavioral Therapies at Modifying the Attitudes of Ekiti State Undergraduates towards Indiscriminate use of Drugs: a paper presented at the Canada International Conference on Education (CICE2012); Canada

[4]. Osakinle, E. O. (2003): The Dynamics of Sexual Behaviours of Female Students in the South Western part of Nigerian Universities. A PhD Dissertation in the Department of Guidance and Counselling, Faculty of Education, Ekiti State University; Ado-Ekiti.

[5]. Shaw, D (2009): Access to Sexual and Reproductive Health for young people: bridging the disconnect between rights and reality. Int. Journal of Gynaecol Obset, 2009; 106(2): 132-136.

[6]. State of World Population (1997): United Nations Population Fund, State of World Population 37.

[7]. The Alan Guttmacher Institute, (1998): Into a new world 40 [hereinafter INTO A NEW WORLD]

[8]. UNFPA (2004); Policy Note on Implementing a Human Rights-Based Approach to Programming in UNFPA. New York. 